\title{
Compressive Strength Prediction of Green Ultra-High Performance Concrete (GUHPC) Based on Adaptive Network-Based Fuzzy Inference System
}

\author{
Angran Xu \\ School of Civil Engineering, Chongqing Jiaotong University, Chongqing, China
}

\begin{abstract}
Green ultra-high performance concrete (GUHPC) is considered to be a new generation of construction materials that adapt to sustainable development and is gradually being used in the fields of bridge reinforcement, house facades, and paving.To improve the efficiency of green ultra-high performance concrete in the experimental stage and to save the component material, the prediction of the 28-day compressive strength of green ultra-high performance concrete has become a challenging task. According to the published literature, the compressive strength of concrete is closely related to the material composition such as cement, fly ash, silica fume, sand, etc. So in this study, 175 groups of related data of GUHPC were collected to form a database, and an artificial neural network system combined with IF-THEN fuzzy rules was utilized to establish a model that could better predict the 28-day compressive strength of GUHPC. Three evaluation indicators, RMSE, $R^{2}$, and MAPE, indicate that the prediction of the compressive strength of green ultra-high performance concrete made by the model is completely reliable. Overall,this study successfully proposes a fuzzy artificial neural network model for predicting the 28-day compressive strength of GUHPC, which provides a viable prediction tool for GUHPC in the experimental stage.
\end{abstract}

Keywords: Green ultra-high performance concrete; Compressive strength prediction; Adaptive network-based fuzzy inference system

\section{Introduction}

In the past decades, the engineering and construction sector has paid increasing attention to environmental issues such as global warming and climate change, and the concrete manufacturing industry is considered to be a source factor of ecological problems. Out of suitability for sustainable development, such as the adoption of laws and the protection of natural resources, playing a significant role in the construction and building materials industry. In recent years, global concrete production has attained 12 billion tons per year, depleting about 1.2 billion tons of silicate cement. Besides, one ton of silicate cement produces over one ton of $\mathbf{C O}_{2}$, which contributes hugely to the greenhouse effect. Therefore, in recent years, scientists have begun researches on green concrete.

The production of cement, a highly energy-intensive material, poses a great challenge for sustainable development. Aghdasi et alnamed green ultra-high performance fiber concrete as G-UHP-FRC (G means green), so this study named green ultra-high performance concrete as GUHPC. Ultra-high performance concrete is nowadays used in a large number of civil engineering fields such as bridge reinforcement, road rehabilitation, house facade reinforcement, tunnels, etc.Ultra-high performance concrete is a cement-based composite material with cement as the base material, fly ash, silica fume, microbeads, and other components as the cementitious supplementary materials, and other stuff like coarse aggregates.As defined, the compressive strength of ultra-high performance concrete is approximately above $150 \mathrm{MPa}$, which is a kind of material with high compatibility, durability, ductility, and toughness. Due to the massive use of cement, which leads to a large amount of carbon dioxide emissions, many researchersare now focusing on environmentally friendly green ultra-high performance concrete. Furthermore, numerous studies have shown that compressive strength is one of the most important mechanical properties of concrete, and since the mechanical properties of ultra-high performance concrete depend on many factors, such as the mix ratio, material quality, water-cement ratio, cement dosage, and the age of the concrete, predicting the compressive strength of the ultra-high performance is becoming a challengingsubject. At present, compressive strength values of concrete are acquired through tests, but the standard compressive strength requires 28days of concrete curing, which is a long waiting process that wastes a lot of time. Based on this, predicting the

ISSN: 0010-8189

(C) CONVERTER 2021

Www.converter-magazine.info 
compressive strength of concrete can decline the number of attempts to fit the ratio, thus reducing the time of test and saving materials.

There are already some research results using artificial intelligence methods and machine learning algorithm modeling to predict the compressive strength of concrete. Predictions of the compressive strength of cement mortars have been made by researchers. Ly et al., Ding et al.made predictions for the strength of manufactured sand concrete. Dao et al.used an adaptive network-based fuzzy inference system (ANFIS) to predict the compressive strength of geopolymer concrete. Other researchers have used deep learning, ANN, evolutionary neural networks, and neural expert systems to predict the compressive strength of recycled aggregate concrete, concrete containing construction waste, and self-compacting concrete containing low ash. Cheng et al.proposed an adaptive fuzzy inference model based on least squares support vector machine (LSSVM) for rubber concrete. Yan et al., Behnood et al., Chou et al.reported support vector machine (SVM) method and regression tree method for predicting elastic modulus of high strength concrete. Sobhani et al.compared the prediction of five slump concrete among the regression model, neural network model, and ANN model, and gained that neural network and ANFIS are more feasible. Nguyen et al.predicted the late compressive strength of concrete based on feedforward neural networks (FNN) and evaluated the performance of the statistical results of 1000 simulations comprehensively, obtaining R, RMSE,MAE for training and testing, which were 0.9861, 2.1501, 1.5650 and 0.9792. The results were better than the classical machine learning algorithms. Chopra et al.used three machine learning techniques for predicting the 28-day, 56-day, and 91-day compressive strength of concrete. The test indicators including ${ }^{2}$ andRMSE of the neural network were 0.9460 and $0.8106,0.9500$ and $0.9100,0.9562$ and 0.9106 , which represents NN'stest performance is the best among the three models. Behnood et al.used the M5P model tree algorithm to predict the compressive strength of normal and high-performance concrete based on 1912 dataand then developed an effective mathematical equation. Bui et al.collected 1133 compressive samples of high-performance concrete from published datasets. They used eight parameters such as cement, water, blast furnace slag, coarse aggregate, fine aggregate, superplasticizer, fly ash, and test age as input quantities and compressive strength as output quantity to simulate in firefly modified ANN model. Finally, they acquiredexcellent model performance by evaluation indicators, R, RMSE, MAE, MAPE of 0.95, 4.85, 3.41, and 11.7 respectively. Öztaş et al.ran a neural network model program in Matlab to predict the compressive strength of high-strength concrete (HSC). They found that the MAPE of compressive strength was less than $1.956208 \%$ and $\mathrm{R}^{2}$ for the test group was $99.93 \%$. Abuodeh et al.selected four materials of ultra-high performance concrete, cement, fly ash, silica fume, and water, as input parameters and combined both neural networks with sequential feature selection (SFS) and NID, calculated $\mathrm{R}^{2}=80.1 \%$ and NMSE=0.012. Zhang et al.used a neural network to predict the compressive strength of ultra-high performance concrete containing SCMs such as fly ash and silica fume. The results showed the high accuracy of the artificial neural network with MAPE and RMSE of $0.0026 \%$ and 4.6564respectively, and $\mathrm{R}^{2}$ of0.9986.

Existing studies have shown that quite reliable results can be obtained for predicting the compressive strength of concrete using artificial intelligence methods and machine learning methods. However, the above methods are more often used to predict general performance concrete and high-performance concrete. For newer material like GUHPC, there are fewer results so far and it is waiting for further study. So based on the current condition of the study, to derive the rule between predicted and experimental values and finally achieve the purpose of predicting the compressive strength of GUHPC, this paper aims to predict the 28-day compressive strength of GUHPC by using the fuzzy artificial neural network method.

\section{Aims and Methodology}

Using the information acquired from the published papers, the work in this paper is organized as follows:First, this paper describes the concept of the fuzzy artificial neural network and the process of building the model.Then the model is trained by the collected data. The performance of the model was evaluated by using the root mean square error (RMSE), correlation coefficient $\left(R^{2}\right)$, and mean absolute percentage error (MAPE), and finally,proved the

ISSN: 0010-8189

(C) CONVERTER 2021 
model applicable to the prediction of compressive strength of GUHPC.

\section{Methods of Modeling}

\section{1 background of artificial neural network}

Artificial neural networks (ANNs) are models based on semi-empirical knowledge of biological nervous systems and are used to simulate the structural behavior of biological nerve cells. A neural network is made up of many processing elements connected by a chain of variable weights and generally consists of neurons in an input layer (also called nodes or processing units), neurons in one or several hidden layers, and neurons in the output layer. The adjacent layers are fully linked to each other by weights, and the input layer neurons receive information from the outside world and pass it to the neurons in the hidden layer without any computation. More specifically, the input layer receives information from the external world, containing a large number of hidden processing units with the output layer[1], and finally, the neurons in the output layer generate network predictions for the external world. First, except for the input layer, each layer of neurons computes a linear combination of the outputs of the neurons in the previous layer, and then adds the deviation, which is the weight. Then, the neurons in the hidden layer apply a nonlinear function to their inputs as an activation function[2].

\subsection{Adaptive neuro-fuzzy inference systems model}

3.2.1 Structure of adaptive neuro-fuzzy inference systems

Adaptive neuro-fuzzy inference systems (ANFIS) are hybrid neuro-fuzzy networks used to modelcomplex systems[2]. ANFIS accomplishes prediction by using fuzzy sets and a code model consisting of IF-THEN fuzzy rules combined with a human-like reasoning process for fuzzy systems[3]. A typical neural network consists of an input, a summation function, a log-sigmoid activation function, and an output. Figure 2 shows the structure of the fuzzy artificial neural network constructed in this paper, with cement (C), fly ash (FA), silica fume (SF), sand (SD), and water (WT) as input parameters being imported into the system. After the interaction between the input parameters, the weights are adjusted by the neural network system and combined with the fuzzy rules, and after two hidden layers of transmission, the final output is the compressive strength (CS).

\subsubsection{Modeling process of adaptive neuro-fuzzy inference systems}

The input is information that enters the cell from other cells in the outside world. The weight is an indication of the degree of influence of the input set or another processing element in the upper layer on that processing element. The summation function is a function that calculates the influence of the inputs and weights on the elements of the process[4,5]. The weighted sum of the components is calculated by the following equation:

$$
(\text { network })_{j}=\sum_{i=1}^{n}\left(w_{i j} x+b\right)
$$

where (network) $)_{j}$ is the output of neurons, $w_{i j}$ is the weight connecting the previous layer to the next layer, $b$ is the bias weight, $n$ is the number of neurons per layer, $f$ is the activation function, and the log-sigmoid activation function in this paper is as follows:

$$
(\text { out })_{j}=f(\text { network })_{j}=\frac{1}{1+e^{-\beta(\text { network })_{j}}}
$$

where $\beta$ is the constant used to control the slope of the semi-linear region,the sigmoid nonlinear function is activated at every layer except the input layer[4]. Using the ANFIS model structure with two input variables as an example, it is assumed that the rules of ANFIS contain Takagi and Sugeno's two IF-THEN rules[6]:

IF $x$ is $A$ and $y$ is $B$, then

ISSN: 0010-8189

(C) CONVERTER 2021 


$$
z=f(x, y)
$$

Figure 1 shows the fuzzy rules, where $A$ and $B$ are the fuzzy sets of the prior terms, $z=f(x, y)$ is the output term. In considering there are two IF-THEN rules in the first-order of Takagi and Sugeno' s model.

Rule1: If $x$ is $A_{1}$ and $y$ is $B_{1}$, then

$$
f_{1}=p_{1} x+q_{1} y+r_{1}
$$

Rule2: If $x$ is $A_{2}$ and $y$ is $B_{2}$, then

$$
f_{2}=p_{2} x+q_{2} y+r_{2}
$$

Layer 1-The output of each node in this layer is expressed as an affiliation function of $A_{i}$

$$
O_{i}^{1}=\mu_{A_{i}}(x)
$$

Layer 2-In this layer, each node multiplies the input signals and sends out the multiplication, the output of each node represents the weight of a rule

$$
w_{i}=\mu_{A_{i}}(y) \times \mu_{B_{i}}(y)
$$

Layer 3- the $i$ th node triggers the ratio of the weight of the $i$ th rule to the sum of the weights of all rules

$$
\overline{w_{i}}=w_{i} /\left(w_{1}+w_{2}+\cdots w_{n}\right)
$$

Layer 4-This layer is the output of Layer 3

$$
O_{i}^{4}=\overline{w_{i}} f_{i}=\overline{w_{i}}\left(p_{i} x+q_{i} y+r_{i}\right)
$$

Layer 5-This layer calculates the total output as a weighted average sum of all signals

$$
O_{i}^{5}=\sum_{i} \bar{w}_{i} f_{i}=\sum_{i} w_{i} f_{i} / \sum_{i} w_{i}
$$

Table 1 shows the properties of the fuzzy artificial neural network constructed in this paper

\section{Data Collection}

The success of the fuzzy artificial neural network model proposed in this paper in predicting the compressive strength of GUHPC depends on the quality of the experimental data for training the fuzzy artificial neural network. The experimental data of GUHPC were collected from published papers [7-9] and used to form the database of this paper. Figure 2 shows the structure of the artificial neural network constructed in this paper, with cement (C), fly ash (FA), silica fume (SF), sand (SD), and water (WT) as input parameters, containing two hidden layers, and compressive strength (CS) as output parameters. Table 2 shows the range of variation for each parameter.

\section{Results and Discussion}

5.1Model training

The Matlab platform is used to build a fuzzy neural network, and the collected GUHPC database is divided into

ISSN: 0010-8189 
two groups: the training set and the prediction set. The model is trained and tested for predictive performance, and the results of prediction are imported into Origin for plotting and analyzing. Table 4 shows the specific data of 145 GUHPCs in the training set and Table 5 shows the specific data of 30 GUHPCs in the prediction set.

5.2Predicted effectiveness

Figure 3 shows the linear fitting results of the training set and the prediction set. Figure 3 a shows the fitted slope of 0.9939 and the fitted correlation coefficient of 0.996 for the simulated results and the experimental values of the training set. Figure $3 b$ shows the fitted slope of 0.9439 and the correlation coefficient of 0.98 for the simulated results and the experimental values of the prediction set. Both the fitted slope and the correlation coefficient are very close to 1 , indicating that the simulated results have low dispersion and high linear correlation with the experimental values, which proves that the model is reliable for prediction.

Figure 4 shows the simulation results of the training and prediction sets compared with the experimental values. From the data comparison in Figure 4b, It can be seen that the larger the value of compressive strength of GUHPC, the higher the prediction accuracy.In addition to this, there are two data points with errors reaching $28.75 \%$ and $25.59 \%$ in Figure 4d, and most of the remaining points have errors below 15\% prediction accuracy. 51 sets of data (about 1/3 of the training set data volume) reach accurate prediction, and the overall prediction performance of the training set is good. Similarly, Figure 4a shows the comparison between the simulation results and the experimental values of the prediction set. Figure $4 \mathrm{c}$ has one data point with an error of $26.97 \%$, and most of the remaining points have an error of less than $15 \%$ prediction accuracy, with 9 sets of data (about $1 / 3$ of the prediction set) achieving accurate prediction, and the prediction set as a whole has good prediction performance.

Figure 5 shows the proposed triangular affiliation function for the input variables using 175 sets of data. The affiliation function consists of 5 input parameters and one output, and the premise parameter subspace is determined by clustering the 175 sets of data. For $f_{c}$, five rules were obtained: If ( $\mathrm{C}$ is $\left.\mathrm{C} m f_{i}\right)$ and (FA is FA $m f_{i}$ )

and $\left(\mathrm{SD}\right.$ is $\mathrm{SD} m f_{i}$ ) and ( $\mathrm{SF}$ is SF $m f_{i}$ ) and ( WT is WT $\left.m f_{i}\right)$ Then ( $f_{c}$ is $\left.f_{c} m f_{i}\right) i=1,2,3,4,5$

\subsection{Modeling performance}

Three experimental metrics, the root mean square error (RMSE), the absolute fraction of variance $\left(R^{2}\right)$, and mean absolute percentage error (MAPE)[10], were used to evaluate the prediction effectiveness of fuzzy artificial neural networks. The root mean square error (RMSE) is calculated using the equation:

$$
R M S E=\sqrt{\frac{1}{p} \sum_{i}\left|t_{i}-o_{i}\right|^{2}}
$$

$p$ is the total number of data in the data set, $t_{i}$ is the true value of the experimental data, and $o_{i}$ is the output value of the model. The absolute fraction of the variance $\left(\mathrm{R}^{2}\right)$ was calculated by the equation:

$$
R^{2}=1-\left[\sum_{i}\left(t_{i}-o_{i}\right)^{2} / \sum_{i}\left(o_{i}\right)^{2}\right]
$$

The mean absolute percentage error (MAPE) was calculated by using equation[5]: 


$$
\operatorname{MAPE}(\%)=\left|\left[\frac{1}{p} \sum_{i=1}^{p} \frac{\left(t_{i}-o_{i}\right)}{o_{i}}\right]\right| \times 100
$$

The absolute difference between a single predicted value and the experimental value was calculated using Error:

$$
\text { Error }=\left|\left(\frac{t_{i}-o_{i}}{o_{i}}\right)\right| \times 100
$$

Table 3 presents the evaluation values of the ANFIS model prediction results, and both the training and prediction sets perform well in terms of metrics. Table 4 lists the experimental values and ANFIS model predictions for each set of GUHPC data.

\section{Conclusions}

In this study, 175 groups of data collected from the open literature that meet the definition of green ultra-high performance concrete (GUHPC) are used to form a database, and 83\% (145 groups) of the data are used as the training set and 17\% (30 groups) of the data are used as the prediction set. Developing an artificial neural network combined with Takagi and Sugeno's first-order hypothetical IF-THEN fuzzy rule for ANIFS model, the following results were achieved in this study:

(1)With C. FA. SF. SD. WT as the five input variables and the ANFIS model as the only output variable, the ANFIS model made very accurate predictions for both the training and prediction sets, indicating that the ANFIS model proposed in this study is fully feasible for predicting the compressive strength of GUHPC $28 \mathrm{~d}$.

(2)Simulations were performed using the training set data, and the evaluation indicators, RMSE, ${ }^{2}$, MAPE, were obtained as 5.3510, 0.9978,4.6103\%. Simulations were performed using the prediction set data, and the evaluation indicators, RMSE, $\mathrm{R}^{2}$, MAPE, were obtained as 2.9952, 0.9950,7.3265\%. The three evaluation indicators prove that the ANFIS model of this study achieves a very accurate prediction of the compressive strength of GUHPC and the performance of the model is excellent.

(3)The overall GUHPC without silica fume in the database exhibits a compressive strength below $100 \mathrm{MPa}$, and for these GUHPC, the prediction error is high compared to other GUHPC with compressive strength above 100 $\mathrm{MPa}$ in the database, with an error of about $15 \%$. This indicates that the model is more applicable to GUHPC with higher compressive strength.

(4)In general, artificial neural networks are more dependent on the size and quality of experimental data. In this study, artificial neural networks are improved by combining them with fuzzy rules, and reliable prediction results are obtained, which shows that the improved method is feasible.

\section{Acknowledgements}

This work was financially supported by National Nature Science Foundation for Young Scientists of China (51908093), Science and Technology Project of the Department of Transport of Hubei Province (2020-2-1-1), Municipal Education Commission Project of Science and Technology Project for Young Scientists of Chongqing (KJQN201900733), General Project of Nature Science Foundation of Chongqing (cstc2020jcyj-msxmX0088). 


\section{References}

[1] B.B. Adhikary, H. Mutsuyoshi, "Prediction of shear strength of steel fiber RC beams using neural networks," Constr. Build. Mater. vol. 20,pp. 801-811, 2006, https://doi.org/10.1016/j.conbuildmat.2005.01.047.

[2] J. Sobhani, M. Najimi, A.R. Pourkhorshidi, T. Parhizkar, "Prediction of the compressive strength of no-slump concrete: A comparative study of regression, neural network and ANFIS models," Constr. Build. Mater. vol. 24,pp. 709-718, 2010, https://doi.org/10.1016/j.conbuildmat.2009.10.037.

[3] A. Mishra, Zaheeruddin, "PAPR Reduction in OFDM Signals: An Adaptive-Network-Based Fuzzy Inference Approach," Wirel. Pers. Commun. vol. 92,pp. 587-601, 2017, https://doi.org/10.1007/s11277-016-3558-4.

[4] I.B. Topçu, M. Saridemir, "Prediction of mechanical properties of recycled aggregate concretes containing silica fume using artificial neural networks and fuzzy logic," Comput. Mater. Sci. vol. 42,pp. 74-82, 2008, https://doi.org/10.1016/j.commatsci.2007.06.011.

[5] I.B. Topçu, M. Saridemir, "Prediction of compressive strength of concrete containing fly ash using artificial neural networks and fuzzy logic," Comput. Mater. Sci. vol. 41,pp. 305-311, 2008, https://doi.org/10.1016/j.commatsci.2007.04.009.

[6] T. Takagi, M. Sugeno, "DERIVATION OF FUZZY CONTROL RULES FROM HUMAN OPERATOR'S CONTROL ACTIONS.," in: IFAC Proc. Ser., IFAC by Pergamon Press, pp. 55-60, 1984, https://doi.org/10.1016/s1474-6670(17)62005-6.

[7] R. Siddique, P. Aggarwal, Y. Aggarwal, "Prediction of compressive strength of self-compacting concrete containing bottom ash using artificial neural networks," Adv. Eng. Softw. vol. 42,pp. 780-786, 2011, https://doi.org/10.1016/j.advengsoft.2011.05.016.

[8] M. Sonebi, "Medium strength self-compacting concrete containing fly ash: Modelling using factorial experimental plans," Cem. Concr. Res. vol. 34,pp. 1199-1208, 2004, https://doi.org/10.1016/j.cemconres.2003.12.022.

[9] A.F. Ghezal, K.H. Khayat, "Optimization of cost-effective self-consolidating concrete," 2016, https://www.researchgate.net/publication/287101060.

[10] D. Van Dao, H. Adeli, H.B. Ly, L.M. Le, V.M. Le, T.T. Le, B.T. Pham, "A sensitivity and robustness analysis of GPR and ANN for high-performance concrete compressive strength prediction using a monte carlo simulation," Sustain. vol. 12, 2020, https://doi.org/10.3390/su12030830. 